\title{
TRACTABILITY OF QUANTIFIED TEMPORAL CONSTRAINTS TO THE MAX
}

\author{
MANUEL BODIRSKY, HUBIE CHEN, AND MICHA£ WRONA
}

\begin{abstract}
A temporal constraint language is a set of relations that are firstorder definable over $(\mathbb{Q} ;<)$. We show that several temporal constraint languages whose constraint satisfaction problem is maximally tractable are also maximally tractable for the more expressive quantified constraint satisfaction problem. These constraint languages are defined in terms of preservation under certain binary polymorphisms. We also present syntactic characterizations of the relations in these languages.
\end{abstract}

Highly Set-Transitive Structures, Constraint Satisfaction Problems, Temporal Constraint Languages, Max-Closed Constraints, Quantified Constraint Satisfaction.

\section{INTRODUCTION}

For a fixed structure $\Gamma$ with finite relational signature $\tau$, the constraint satisfaction problem of $\Gamma$, denoted by $\operatorname{CSP}(\Gamma)$, is the problem of deciding whether a given sentence of the form $\exists x_{1} \ldots \exists x_{n}\left(\psi_{1} \wedge \cdots \wedge \psi_{m}\right)$ is true in $\Gamma$; here, each $\psi_{i}$ is an atomic $\tau$-sentence, that is, of the form $R\left(y_{1}, \ldots, y_{k}\right)$ for $R \in \tau$ and $y_{1}, \ldots, y_{k} \in\left\{x_{1}, \ldots, x_{n}\right\}$. The given sentence is called an instance of $\operatorname{CSP}(\Gamma)$, and the formulas $\psi_{i}$ are called the constraints of the instance. The set of relations of $\Gamma$ is called the constraint language of $\Gamma$.

A very active research program attempts to classify the computational complexity of $\operatorname{CSP}(\Gamma)$ for all finite structures $\Gamma$; see e.g. the collection of survey articles [16]. Particularly interesting are structures $\Gamma$ that are maximally tractable; the idea is that those structures $\Gamma$ are the structures that sit at the frontier between the easy and the hard CSPs. The notion of maximal tractability also applies to structures $\Gamma$ with an infinite relational signature. We say that $\Gamma$ is maximally tractable for the CSP with respect to a class $\mathcal{C}$ of relations over the same domain (and in this case we also say that the constraint language of $\Gamma$ is maximally tractable) if

- for every structure $\Gamma^{\prime}$ whose constraint language is finite and contained in the constraint language of $\Gamma$, the problem $\operatorname{CSP}\left(\Gamma^{\prime}\right)$ is in $\mathrm{P}$, and

- for every set of relations $\mathcal{R} \subseteq \mathcal{C}$ that properly contains the constraint language of $\Gamma$ there exists a structure $\Gamma^{\prime \prime}$ with a finite constraint language that is contained in $\mathcal{R}$ such that $\operatorname{CSP}\left(\Gamma^{\prime \prime}\right)$ is NP-hard.

Surprisingly often, when $\Gamma$ is maximally tractable for the CSP with respect to a class of relations, the more expressive quantified constraint satisfaction problem for $\Gamma$, denoted by $\mathrm{QCSP}(\Gamma)$, can be solved in polynomial time as well. The problem

The research leading to the results presented here has also received funding from the European Research Council under the European Community's Seventh Framework Program (FP7/2007-2013 Grant Agreement no. 257039.

The author is supported by the Swedish Research Council (VR) under grant 621-2012-3239. 
$\operatorname{QCSP}(\Gamma)$ is defined analogously to $\operatorname{CSP}(\Gamma)$, with the difference that, in addition to existential quantification, universal quantification is also permitted in the input. That is, an instance of $\mathrm{QCSP}(\Gamma)$ is a sentence of the form $Q_{1} v_{1} \ldots Q_{n} v_{n}\left(\psi_{1} \wedge \cdots \wedge\right.$ $\left.\psi_{m}\right)$, for $Q_{1}, \ldots, Q_{n} \in\{\exists, \forall\}$, to be evaluated in $\Gamma$.

While one might expect that $\operatorname{QCSP}(\Gamma)$ is typically harder than $\operatorname{CSP}(\Gamma)$, for the following structures $\Gamma$ both $\operatorname{CSP}(\Gamma)$ and $\operatorname{QCSP}(\Gamma)$ are polynomial-time decidable:

(1) 2-element structures $\Gamma$ preserved by min or by $\max [20$;

(2) 2-element structures $\Gamma$ preserved by the majority operation 1;

(3) 2-element structures $\Gamma$ preserved by the minority operation [15;

(4) structures $\Gamma$ preserved by a near-unanimity operation [13;

(5) structures $\Gamma$ preserved by a Maltsev operation [9;

(6) structures $\Gamma$ that contain a unary relation $U$ for every subset of its domain, and $\operatorname{CSP}(\Gamma)$ is tractable [5].

See [14] for a survey on the state of the art of complexity classification for $\mathrm{QCSP}(\Gamma)$ for finite structures $\Gamma$.

Maximal tractability of a structure $\Gamma$ for the QCSP is defined analogously to maximal tractability of $\Gamma$ for the CSP. Note that if $\Gamma$ is maximally tractable for the CSP with respect to some class of relations $\mathcal{C}$, and if for all structures $\Gamma^{\prime}$ whose constraint language is finite and contained in the constraint language of $\Gamma$ the problem $\operatorname{QCSP}\left(\Gamma^{\prime}\right)$ is in $\mathrm{P}$ as well, then $\Gamma^{\prime}$ is maximally tractable for the QCSP with respect to $\mathcal{C}$ as well. This is for instance the case for all the maximally tractable structures $\Gamma$ that appear in (1)-(6) above (each of those 6 items contains structures $\Gamma$ that are maximally tractable for the CSP with respect to the class of all relations over the same domain). If structures that are maximally tractable for the CSP are also maximally tractable for the QCSP, then this is interesting because it tells us that in this case the restriction to only existential quantifiers does not make a hard problem easy.

In this article we study this transfer of maximal tractability for the CSP to maximal tractability for the QCSP for structures $\Gamma$ with an infinite domain. One of the best-understood classes of infinite structures is the class of all highly set-transitive structures; these are the structures $\Gamma$ with the property that for all finite subsets $A, B$ of equal size, there exists an automorphism of $\Gamma$ which sends $A$ to $B$. By a theorem due to Cameron [10, this is the case if and only if $\Gamma$ is isomorphic to a structure with domain $\mathbb{Q}$ whose relations are first-order definable over $(\mathbb{Q} ;<)$, i.e., for each relation $R$ of $\Gamma$ there is a first-order formula that defines $R$ over $(\mathbb{Q} ;<)$ (without parameters). In the context of constraint satisfaction, the constraint languages of highly set-transitive structures have also been called temporal constraint languages, since many problems in qualitative temporal reasoning can be formulated as $\operatorname{CSP}(\Gamma)$ for such structures $\Gamma$ (see 7 , 8, for examples). We refer to a relation having a first-order definition in $(\mathbb{Q} ;<)$ as a temporal relation. From now on, maximal tractability will be with respect to the class of all temporal relations over $\mathbb{Q}$.

The class of temporal constraint languages is very rich, and often provides examples and counterexamples for CSPs of infinite structures $\Gamma$. For example, for finite structures $\Gamma$ it is known that $\operatorname{CSP}(\Gamma)$ can be solved by Datalog if and only if the core of $\Gamma$ expanded by constants does not interpret primitively positively the structure $\left(\mathbb{Z}_{p} ;\{(x, y, z) \mid x-y+z=1\}\right)$; this follows from the main result in [2] in combination with [17]. The same statement is false already for 
the simple structure $(\mathbb{Q} ;\{(x, y, z) \mid x>y \vee x>z\})$, which does not interpret $\left(\mathbb{Z}_{p} ;\{(x, y, z) \mid x-y+z=1\}\right)$ primitively positively with constant $\{$, but which cannot be solved by Datalog; see 8 . The list of phenomena that are specific to infinite-domain constraint satisfaction and that are exemplified already for temporal constraint languages can be prolonged easily; we refer to [3] for more detail.

The complexity of $\operatorname{CSP}(\Gamma)$ has been completely classified for structures $\Gamma$ with a temporal constraint language (due to [7]; also see [3]). In this paper we visit some of the CSP maximally tractable temporal constraint languages and study their QCSP. For the languages that we study, we show that even the QCSP is polynomial-time tractable. Our results are as follows.

- When all relations of $\Gamma$ are preserved by the operation $(x, y) \mapsto \min (x, y)$, then $\operatorname{QCSP}(\Gamma)$ is in $\mathrm{P}$.

- When all relations in $\Gamma$ are preserved by the operation $\mathrm{mx}$ (introduced in [7), then $\operatorname{QCSP}(\Gamma)$ is in P. As with min, the operation $m x$ is binary and commutative; we repeat the definition in Section 3.3 .

We complement those results by giving intuitive syntactic descriptions of the temporal relations that are preserved by min, and likewise of those that are preserved by $\mathrm{mx}$. That is, we present a class of syntactically restricted first-order formulas with the property that a temporal relation is preserved by min if and only if it has a definition by a formula from the class, and likewise for mx. Those characterizations are important since they give a more explicit description of the allowed constraints in the respective QCSPs. A similar syntactic description of relations over finite domains preserved by min can be found in [19].

The results that we present for temporal constraint languages preserved by min also hold in analogous form for those that are preserved by max. The operations min and $\max$ are dual in the following sense: $\max (x, y)=-\min (-x,-y)$. Similarly, the operation $\mathrm{mx}$ has the dual operation $(x, y) \mapsto-\operatorname{mx}(-x,-y)$, and again the results that we have obtained for $\mathrm{mx}$ also hold analogously for the dual of $\mathrm{mx}$.

\section{Temporal Constraint Languages}

Let $f: D^{k} \rightarrow D$ be a function. When $t^{1}=\left(t_{1}^{1}, \ldots, t_{m}^{1}\right), \ldots, t^{k}=\left(t_{1}^{k}, \ldots, t_{m}^{k}\right) \in$ $D^{m}$, then $f\left(t^{1}, \ldots, t^{k}\right)$ denotes the tuple $\left(f\left(t_{1}^{1}, \ldots, t_{1}^{k}\right), \ldots, f\left(t_{m}^{1}, \ldots, t_{m}^{k}\right)\right)$ from $D^{m}$ that we obtain by applying $f$ componentwise. A function $f: D^{k} \rightarrow D$ preserves a relation $R \subseteq D^{m}$ if for all $t^{1}, \ldots, t^{k} \in R$ it holds that $f\left(t^{1}, \ldots, t^{k}\right) \in R$. If $f$ preserves all relations of a structure $\Gamma$, we say that $f$ is a polymorphism of $\Gamma$.

Example. The operation min: $\mathbb{Q}^{2} \rightarrow \mathbb{Q}$ that maps two rational numbers to the minimum of the two numbers is a polymorphism of the temporal constraint language $(\mathbb{Q} ; \leq,<)$, but not of the temporal constraint language $(\mathbb{Q} ; \neq)$, since it maps for instance the tuples $(1,0) \in \neq$ and $(0,1) \in \neq$ to $(0,0) \notin \neq$.

See Figure 1 for an illustration of the operation min. In diagrams for binary operations $f$ as in Figure 3, we draw a directed edge from $(a, b)$ to $\left(a^{\prime}, b^{\prime}\right)$ if $f(a, b)<$ $f\left(a^{\prime}, b^{\prime}\right)$. Unoriented lines in rows and columns of picture for an operation $f$ relate pairs of values that get the same value under $f$.

\footnotetext{
${ }^{1}$ Since it has the polymorphism $f(x, y)=\min (x, y)$ satisfying $\forall x, y(f(x, y)=f(y, x))$; see [3] for an introduction to primitive positive interpretations and the related universal algebra.
} 


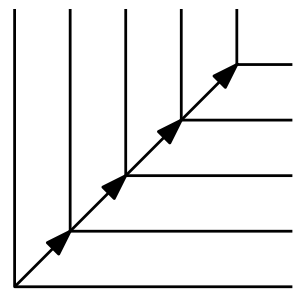

FIGURE 1. Illustration of the operation min.

A non-trivial relation that is preserved by min is the ternary relation defined by the formula $x_{1}>x_{2} \vee x_{1}>x_{3}$, and the ternary relation $U$ defined as follows.

$$
\begin{aligned}
U(x, y, z) & \equiv(x=y \wedge y<z) \\
& \vee(x=z \wedge z<y) \\
& \vee(x=y \wedge y=z)
\end{aligned}
$$

Another important operation for temporal constraint satisfaction is the binary operation ' $m x$ ', which is defined as follows [7.

$$
\operatorname{mx}(x, y):= \begin{cases}\alpha(\min (x, y)) & \text { if } x \neq y \\ \beta(x) & \text { if } x=y\end{cases}
$$

where $\alpha$ and $\beta$ are unary operations that preserve $<$ such that $\alpha(x)<\beta(x)<$ $\alpha(x+\varepsilon)$ for all $x \in \mathbb{Q}$ and all $0<\varepsilon \in \mathbb{Q}$ (see Figure 2). It is not difficult to show that such operations $\alpha, \beta$ do exist; see [7].

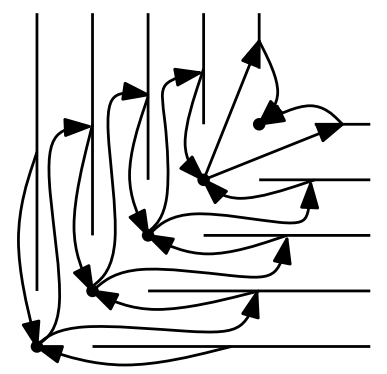

FiguRE 2. Illustration of the operation mx.

Note that $\mathrm{mx}$ does not preserve the relation $\leq$. It also does not preserve the relation $U$ introduced above. An example of a relation that is preserved by $\mathrm{mx}$ is the ternary relation $X$ defined as follows.

$$
\begin{array}{r}
X(x, y, z) \equiv(x=y \wedge y<z) \\
\vee(x=z \wedge z<y) \\
\vee(y=z \wedge y<x)
\end{array}
$$

Finally, let pp be an arbitrary binary operation on $\mathbb{Q}$ such that $\operatorname{pp}(a, b) \leq$ $\operatorname{pp}\left(a^{\prime}, b^{\prime}\right)$ iff one of the following cases applies: 
- $a \leq 0$ and $a \leq a^{\prime}$

- $0<a, 0<a^{\prime}$, and $b \leq b^{\prime}$.

Clearly, such an operation exists. For an illustration, see the left diagram in Figure 3. The right diagram of Figure 3 is an illustration of the dual-pp operation. The name of the operation pp is derived from the word 'projection-projection', since the operation behaves as a projection to the first argument for negative first argument, and a projection to the second argument for positive first argument.
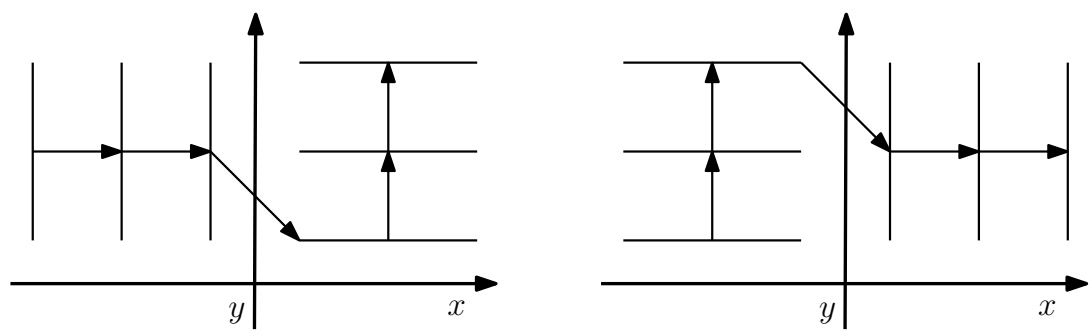

Figure 3. A visualization of pp (left) and dual-pp (right).

The following two propositions have been shown in [7].

Proposition 2.1 ([7). Let $R_{1}, \ldots, R_{k}$ be temporal relations that are preserved by min (respectively, $m x)$. Then $\operatorname{CSP}\left(\mathbb{Q} ; R_{1}, \ldots, R_{k}\right)$ is in $P$.

Proposition $2.2([7)$. Let $R$ be a relation with a first-order definition in $(\mathbb{Q} ;<)$ that is not preserved by min (respectively, $m x$ ). Then there exists a finite set of temporal relations $R_{1}, \ldots, R_{k}$ preserved by min (respectively, $m x$ ) such that $\operatorname{CSP}\left(\mathbb{Q} ; R, R_{1}, \ldots, R_{k}\right)$ is NP-hard.

A straightforward corollary of Proposition 2.1 and Proposition 2.2 is that the sets of temporal relations preserved by min and by $\mathrm{mx}$, respectively, are maximally tractable constraint languages.

The set of temporal relations that is preserved by min is a proper subset of the set of temporal relations that is preserved by pp (see [7]); e.g., the relation $\left\{(x, y, z) \in \mathbb{Q}^{3} \mid(x=y \wedge y<z) \vee(x>y \wedge y=z)\right\}$ is preserved by pp, but not by min and not by $\mathrm{mx}$. Indeed, the CSP for the temporal constraint language that just contains this ternary relation is NP-hard.

\section{Syntactic Characterizations}

It is known that the structure $(\mathbb{Q} ;<)$ has quantifier-elimination 18 , that is, for every first-order formula $\phi$ there exists a quantifier-free formula that is equivalent to $\phi$ over $(\mathbb{Q} ;<)$ (we always allow equality $=$ in our formulas). We will call such a quantifier-free formula over the signature $\{<\}$ a temporal formula. For the sake of presentation, we write temporal formulas using all symbols in $\{<\rangle,, \leq, \geq,=, \neq\}$. Let $\phi_{R}$ be a temporal formula that defines a temporal relation $R$. For a tuple $t \in R$ and a variable $v$ occurring in $\phi_{R}$, we write $t(v)$ to indicate the value in $t$ corresponding to the variable $v$.

It is also well-known that $(\mathbb{Q} ;<)$ is a homogeneous structure 21], that is, every isomorphism between finite substructures extends to an automorphism. The set 
of automorphisms of $(\mathbb{Q} ;<)$ will be denoted by $\operatorname{Aut}(\mathbb{Q} ;<)$. A set of the form $\left\{\alpha\left(t_{1}\right), \ldots, \alpha\left(t_{k}\right) \mid \alpha \in \operatorname{Aut}(\mathbb{Q} ;<)\right\}$ is called an orbit of $k$-tuples (the orbit of $\left.\left(t_{1}, \ldots, t_{k}\right)\right)$. It is well known that every $k$-ary temporal relation is a union of orbits of $k$-tuples.

Let $\phi_{1}$ and $\phi_{2}$ be two temporal formulas. We say that $\phi_{1}$ entails $\phi_{2}$ if and only if $(\mathbb{Q} ;<) \models \forall v_{1}, \ldots, v_{n}\left(\phi_{1} \Rightarrow \phi_{2}\right)$. We also say that an $n$-ary temporal relation $R$ entails $\phi$ if and only if $(\mathbb{Q} ;<) \models \forall v_{1}, \ldots, v_{n}\left(\phi_{R}\left(v_{1}, \ldots v_{n}\right) \Rightarrow \phi\right)$, where $\phi_{R}$ is a temporal formula defining $R$. We will use the following fact, which follows directly from the definition of entailment.

Observation 3.1. Let $\phi_{R}:=\Phi \wedge \psi$ be a temporal formula defining a temporal relation $R$ and $\psi^{\prime}$ a temporal formula entailed by $R$ which entails $\psi$. Then $\phi_{R}^{\prime}:=$ $\Phi \wedge \psi^{\prime}$ also defines $R$.

\subsection{Characterization of pp-closed Relations.}

Theorem 3.2. A temporal relation $R$ is preserved by $\mathrm{pp}$ if and only if it can be defined as a conjunction of clauses of the form

$$
x \neq y_{1} \vee \cdots \vee x \neq y_{k} \vee x \geq z_{1} \vee \cdots \vee x \geq z_{l},
$$

where it is permitted that $l=0$ or $k=0$, in which cases the clause is a disjunction of disequalities or contains no disequalities, respectively.

To provide the proof we fix $n$, and consider a temporal relation of arity $n$. All the temporal formulas considered in the proof of Theorem 3.2 are meant to have all variables in $\left\{v_{1}, \ldots, v_{n}\right\}$. In particular we take advantage of temporal formulas of the form:

$$
\neg\left(x_{1} \circ_{1} x_{2} \wedge \cdots \wedge x_{k-1} \circ_{k-1} x_{k}\right),
$$

where $x_{1}, \ldots, x_{k} \in\left\{v_{1}, \ldots, v_{n}\right\}$ are pairwise different and every $o_{i}$ is in $\{<,=\}$. We focus on formulas of the form (2) that satisfy an extra condition that we call the unambiguity condition which states that if $\left(v_{i}=v_{j}\right)$ occurs in $\phi$, then $i<j$. Let $\Phi_{R}$ be the set containing all temporal formulas $\phi$ over variables $\left\{v_{1}, \ldots, v_{n}\right\}$ of the form $(2)$ entailed by $R$ that satisfy the unambiguity condition. We will now argue that $\bigwedge \Phi_{R}$ defines $R$. Observe first that for every orbit of $n$-tuples in $\operatorname{Aut}(\mathbb{Q} ;<)$, there is the negation $\phi$ of a formula of the form (2) that defines this orbit. We can certainly assume that $\phi$ is over variables $\left\{v_{1}, \ldots, v_{n}\right\}$ and satisfies the unambiguity condition. On the other hand, to see that every such $\phi$ defines exactly one orbit observe that any two tuples $t:=\left(t_{1}, \ldots, t_{n}\right)$ and $t^{\prime}:=\left(t_{1}^{\prime}, \ldots, t_{n}^{\prime}\right)$ that are in the relation defined by $\phi$ induce isomorphic substructures in $(\mathbb{Q} ;<)$. By the homogeneity of $(\mathbb{Q} ;<)$, an isomorphism between such substructures can be extended to an automorphism of $(\mathbb{Q} ;<)$. It follows that every orbit may be defined by the negation $\phi$ of a formula of the form $(2)$. We are now ready to prove that $R^{\prime}$ defined by $\bigwedge \Phi_{R}$ is equal to $R$. By the definition of $R^{\prime}$ we have that $R \subseteq R^{\prime}$. On the other hand if there is an orbit in $\operatorname{Aut}(\mathbb{Q} ;<)$ which is not in $R$, then an appropriate formula of the form (2) is entailed by $R$. Thus $R^{\prime}$ does not contain the orbit as well. Hence we obtain that $\bigwedge \Phi_{R}$ defines $R$.

Finally, we define an order on $\Phi_{R}$. For $\phi_{1}, \phi_{2} \in \Phi_{R}$ of the form $\neg\left(x_{1} \circ_{1} x_{2} \wedge\right.$ $\left.\cdots \wedge x_{k-1} \circ_{k-1} x_{k}\right)$ and $\neg\left(y_{1} \circ_{1} y_{2} \wedge \cdots \wedge y_{l-1} \circ_{l-1} y_{l}\right)$, respectively, we will say that $\phi_{1}$ is less than $\phi_{2}$, in symbols $\phi_{1} \preceq \phi_{2}$, if $x_{1}, \ldots, x_{k}$ is a subsequence of $y_{1}, \ldots, y_{l}$ and $\phi_{1}$ entails $\phi_{2}$. It is easy to see that this relation is reflexive and transitive. By 
the definition of $\Phi_{R}$, we have that two formulas $\phi_{1}, \phi_{2} \in \Phi_{R}$ with the same set of variables entail each other if and only if they are the same formula. Hence, $\left(\Phi_{R} ; \preceq\right)$ is also antisymmetric, and we have defined a partial order on $\Phi_{R}$. Let $\Phi_{R}^{m}$ be the set of minimal elements of $\left(\Phi_{R} ; \preceq\right)$. By Observation 3.1 it holds that $\bigwedge \Phi_{R}^{m}$ defines $R$.

Proof. To prove the right-to-left implication, it is certainly enough to show that every relation $R_{C}$ defined by a single clause $C$ of the form (1) is preserved by pp. Assume on the contrary that there are $t_{1}, t_{2} \in R_{C}$ but $t_{3}=\operatorname{pp}\left(t_{1}, t_{2}\right)$ is not in $R_{C}$. It implies that $t_{3}(x)=t_{3}\left(y_{1}\right)=\cdots=t_{3}\left(y_{k}\right)$, and hence by the definition of pp it holds that $t_{1}(x)=t_{1}\left(y_{1}\right)=\cdots=t_{1}\left(y_{k}\right)$ or $t_{2}(x)=t_{2}\left(y_{1}\right)=\cdots=t_{2}\left(y_{k}\right)$. In the first case, $t_{1}(x) \leq 0$ and since for at least one $z_{j}$ we have that $t_{1}\left(z_{j}\right) \leq t_{1}(x)$, it follows that $t_{3}\left(z_{j}\right) \leq t_{3}(x)$. Otherwise, we have that $t_{1}(x)>0$. Again, for at least one $z_{j}$ it holds that $t_{2}\left(z_{j}\right) \leq t_{2}(x)$. It follows that $t_{3}\left(z_{j}\right) \leq t_{3}(x)$. Thus, every clause of the form (1) is preserved by pp.

We now show that every temporal relation $R$ preserved by pp may be defined as a conjunction of clauses of the desired form. Recall that $\bigwedge \Phi_{R}^{m}$ defines $R$. We will obtain the desired definition of $R$ by replacing every member $\phi$ of $\Phi_{R}^{m}$, which is of the form (2), with a clause of the form (1). If $\phi$ is $\neg\left(x=y_{1} \wedge \cdots \wedge y_{k-1}=y_{k}\right)$, then we replace it by $\left(x \neq y_{1} \vee \cdots \vee x \neq y_{k}\right)$. Since these formulas are equivalent, the transformation does not change the defined relation.

Otherwise, $\phi$ contains at least one occurrence of $<$ and hence we can assume that $\phi$ is of the form:

$$
\neg\left(x=y_{1} \wedge \cdots \wedge y_{k-1}=y_{k} \wedge y_{k}<z_{1} \wedge z_{1} \circ_{1} z_{2} \wedge \cdots \wedge z_{l-1} \circ_{l-1} z_{l}\right),
$$

where every $\circ_{i}$ is in $\{=,<\}$. Here we will consider two cases. The first is where $R$ contains no tuple $t$ satisfying $t(x)=t\left(y_{1}\right)=\cdots=t\left(y_{k}\right)<t\left(z_{i}\right)$ for all $i \in[l]$. Observe that in this case $R$ entails $\psi$ equal to $\left(x \neq y_{1} \vee \cdots \vee x \neq y_{k} \vee x \geq z_{1} \vee \cdots \vee x \geq\right.$ $\left.z_{l}\right)$. It is easy to verify that $\psi$ entails $\phi$. Hence, by Observation 3.1, it follows that we can safely replace $\phi$ by $\psi$ in $\Phi_{R}^{m}$.

If $R$ contains a tuple $t$ satisfying $t(x)=t\left(y_{1}\right)=\cdots=t\left(y_{k}\right)<t\left(z_{i}\right)$ for all $i \in[l]$, then, as we show, $R$ is not preserved by pp. This leads to a contradiction with the assumption. Consider a formula $\theta$ equal to $\neg\left(z_{1} \circ_{1} z_{2} \wedge \cdots \wedge z_{l-1} \circ_{l-1} z_{l}\right)$. We have $\theta \preceq \phi$. Since $\phi$ is in $\Phi_{R}^{m}$, it follows that $\theta$ is not in $\Phi_{R}$, and hence not entailed by $R$. It implies that $R$ contains a tuple $t_{1}$ satisfying $\left(z_{1} \circ_{1} z_{2} \wedge \cdots \wedge z_{l-1} \circ_{l-1} z_{l}\right)$. Let $\alpha$ be automorphisms of $(\mathbb{Q} ;<)$ such that $\alpha(t(x))<0<\alpha\left(t\left(z_{i}\right)\right)$ for all $i \in[l]$. Then $t_{2}=\operatorname{pp}\left(\alpha(t), t_{1}\right)$ satisfies $\neg \phi$. Since $\phi$ is entailed by $R$, it follows $t_{2} \notin R$, and thus that $R$ is not preserved by pp.

\subsection{Characterization of min-closed Relations.}

Theorem 3.3. A temporal relation $R$ is preserved by $\min$ if and only if it can be defined as a conjunction of clauses of the form

$$
x \circ_{1} z_{1} \vee \cdots \vee x \circ_{l} z_{l},
$$

where for all $i \in[l]$, we have that $\circ_{i}$ is in $\{\geq,>\}$.

Proof. Observe that to prove the left-to-right implication, it is enough to show that every relation $R_{C}$ defined by a single clause $C$ of the form (3) is preserved by min. Let $t_{1}, t_{2}$ be some tuples in $R$ and $t_{3}=\min \left(t_{1}, t_{2}\right)$. Then $t_{1}, t_{2}$ satisfy $x \circ_{i} z_{i}$, and $x \circ_{j} z_{j}$ for some $i, j \in[l]$, respectively. We consider two cases. Assume first that 
$t_{1}\left(z_{i}\right)=t_{2}\left(z_{j}\right)$. Here, it is easy to see that $t_{3}(x) \geq t_{3}\left(z_{i}\right)$ and $t_{3}(x) \geq t_{3}\left(z_{j}\right)$. Thus if either $\circ_{i}$ or $\circ_{j}$ is $\geq$, then we are done. Otherwise, it holds that $t_{1}(x)>t_{1}\left(z_{i}\right)$ and $t_{2}(x)>t_{2}\left(z_{j}\right)$. It follows that $t_{3}(x)$ is greater than both $t_{3}\left(z_{i}\right)$ and $t_{3}\left(z_{j}\right)$. This settles the case where $t_{1}\left(z_{i}\right)=t_{2}\left(z_{j}\right)$. Assume now without loss of generality that $t_{1}\left(z_{i}\right)<t_{2}\left(z_{j}\right)$. Observe that $t_{3}\left(z_{i}\right) \leq t_{3}(x)$. Thus we are done when $\circ_{i}$ is $\leq$. Otherwise we have that $t_{1}\left(z_{i}\right)<t_{1}(x)$ and that $t_{1}\left(z_{i}\right)<t_{2}\left(z_{j}\right) \leq t_{2}(x)$. It follows that $t_{3}\left(z_{i}\right)<t_{3}(x)$. It completes the proof of the left-to-right implication.

We now turn to prove the reverse implication. Assume that there is some relation $R$ preserved by min that cannot be defined by a conjunction of clauses of the form (3). By Lemma 23 in 7 , we have that every relation preserved by min is also preserved by pp. Hence, by Theorem 3.2 , the relation $R$ may be defined as a conjunction of clauses of the form

$$
x \neq y_{1} \vee \cdots \vee x \neq y_{k} \vee x \circ_{1} z_{1} \vee \cdots \vee x \circ_{l} z_{l},
$$

where $\circ_{i} \in\{>, \geq\}$ for every $i \in[l]$. Consider the set of such definitions of $R$ with a minimal number of disequalities and from this set choose one with a minimal number of literals. Denote that formula by $\phi_{R}$.

If $\phi_{R}$ does not have any disequalities, then we are done. Otherwise it contains a clause $C$ of the form (4) with at least one disequality $\left(x \neq y_{1}\right)$. Since $\phi_{R}$ does not contain any unnecessary literals, there is a tuple in $R$ that falsifies all literals in $C$ except for $\left(x \neq y_{1}\right)$. Suppose that $R$ contains both tuples $t_{1}, t_{2}$ that falsify all literals in $C$ except for $\left(x \neq y_{1}\right)$ and satisfy $\left(x<y_{1}\right)$ and $\left(x>y_{1}\right)$, respectively. Let $\alpha_{1}$ be an automorphism of $(\mathbb{Q} ;<)$ that sends $\left(t_{1}(x), t_{1}\left(y_{1}\right)\right)$ to $(0,1)$, and $\alpha_{2}$ an automorphism of $(\mathbb{Q} ;<)$ that sends $\left(t_{2}(x), t_{2}\left(y_{1}\right)\right)$ to $(1,0)$. Observe that $t_{3}=$ $\min \left(\alpha_{1}\left(t_{1}\right), \alpha_{2}\left(t_{2}\right)\right)$ satisfies $\left(x=y_{1}\right)$. Since min preserves $=, \leq$, and $<$, we have that $t_{3}$ falsifies all literals in $C$, hence we have a contradiction with the fact that $R$ is preserved by min. It follows that $R$ does not have either $t_{1}$ or $t_{2}$. If $R$ does not contain $t_{1}$, then in $\phi_{R}$ we can replace $C$ by $\left(x>y_{1} \vee x \neq y_{2} \vee \cdots \vee x \neq\right.$ $y_{k} \vee x \circ_{1} z_{1} \vee \cdots \vee x \circ_{l} z_{l}$ ) obtaining a definition of $R$ guaranteed by Theorem 3.2 with a smaller number of disequalities. From now on we can therefore assume that $R$ contains $t_{1}$.

Consider now the formula $\phi_{R}^{\prime}$ obtained from $\phi_{R}$ by replacing the clause $C$ by the clause $C^{\prime}:=\left(y_{1} \neq x \vee y_{1} \neq y_{2} \vee \cdots \vee y_{1} \neq y_{k} \vee y_{1} \circ_{1} z_{1} \vee \cdots \vee y_{1} \circ_{l} z_{l}\right)$. Observe that $C$ and $C^{\prime}$ entail each other. Hence $\phi_{R}^{\prime}$ also defines $R$. Consider $C^{\prime}$ as a part of $\phi_{R}^{\prime}$ and observe that no literal from $C^{\prime}$ can be removed. Indeed, the new definition would have less disequlities or the same number of disequalities and less literals than $\phi_{R}$. Thus $R$ contains a tuple $t^{\prime}$ that satisfies all disjuncts of $C^{\prime}$ except for $\left(y_{1} \neq x\right)$. As in the previous papragraph, we argue that $R$ cannot have both tuples $t_{1}^{\prime}$, and $t_{2}^{\prime}$ that falsify all literals in $C^{\prime}$ except for $\left(x \neq y_{1}\right)$ and satisfy $\left(y_{1}<x\right)$ and $\left(y_{1}>x\right)$, respectively. If $R$ does not contain $t_{1}^{\prime}$, then in $\phi_{R}^{\prime}$ we can replace $C^{\prime}$ by $\left(y_{1}>x \vee y_{1} \neq y_{2} \vee \cdots \vee y_{1} \neq y_{k} \vee y_{1} \circ_{1} z_{1} \vee \cdots \vee y_{1} \circ_{l} z_{l}\right)$ obtaining a definition of $R$ in the form guaranteed by Theorem 3.2 with a smaller number of disequalities. Thus, we can assume that $R$ contains $t_{1}^{\prime}$.

Now, suppose towards the contradiction that $R$ has both: $t_{1}$ that falsifies all disjuncts of $C$ except for $\left(x \neq y_{1}\right)$ and satisfies $\left(x<y_{1}\right)$; and $t_{1}^{\prime}$ that falsifies all disjuncts of $C^{\prime}$ except for $\left(y_{1} \neq x\right)$ and satisfies $\left(y_{1}<x\right)$. Let $\alpha, \alpha^{\prime}$ be automorphisms of $\operatorname{Aut}(\mathbb{Q} ;<)$ such that $\alpha$ sends $\left(t_{1}(x), t_{1}\left(y_{1}\right)\right)$ to $(0,1)$ and $\alpha^{\prime}$ sends $\left(t_{1}^{\prime}(x), t_{1}^{\prime}\left(y_{1}\right)\right)$ to $(1,0)$. To complete the proof we will show that $t_{4}=\min \left(\alpha\left(t_{1}\right), \alpha^{\prime}\left(t_{1}^{\prime}\right)\right)$ falsifies all disjuncts of $C$. Since both $t_{1}$ and $t_{1}^{\prime}$ are in $R$, we obtain a contradiction to 
the assumption that $R$ is preserved by min. Since $\alpha\left(t_{1}(x)\right)=\alpha\left(t_{1}\left(y_{2}\right)\right)=\cdots=$ $\alpha\left(t_{1}\left(y_{k}\right)\right)=0$ and $\alpha^{\prime}\left(t_{1}^{\prime}\left(y_{1}\right)\right)=\alpha^{\prime}\left(t_{1}^{\prime}\left(y_{2}\right)\right)=\cdots=\alpha^{\prime}\left(t_{1}^{\prime}\left(y_{k}\right)\right)=0$, it follows that $t_{4}(x)=t_{4}\left(y_{1}\right)=\cdots=t_{4}\left(y_{k}\right)=0$ and hence $t_{4}$ falsifies all disequalities in $C$. Now, the clause $C$ contains a disjunct $\left(x \circ_{i} z_{i}\right)$ with $\circ_{i} \in\{>, \geq\}$ and $i \in[k]$ if and only if $C^{\prime}$ contains $\left(y_{1} \circ_{i} z_{i}\right)$. Assume that $\circ_{i}$ is $>$. The same argument will work for $\geq$. Observe that $\alpha\left(t_{1}\right)$ satisfies $\left(x \leq z_{i}\right)$ and sends $x$ to 0 ; and that $\alpha^{\prime}\left(t_{1}^{\prime}\right)$ satisfies $\left(y_{1} \leq z_{i}\right)$ and sends $y_{1}$ to 0 . It follows that $t_{4}(x) \leq 0 \leq t_{4}\left(z_{i}\right)$. Thus $t_{4}$ falsifies $\left(x<z_{i}\right)$ and we are done.

Example. According to Theorem 3.3 , the relation $U$ from Section 2 can be defined by a conjunction of clauses of the form 3 . Indeed, observe that $((x \geq$ $y \vee x \geq z) \wedge y \geq x \wedge z \geq x$ ) defines $U$.

3.3. Characterization of mx-closed relations. Let us say that a boolean relation $S \subseteq\{0,1\}^{n}$ is near-affine if it holds that $S \cup\{(1, \ldots, 1)\}$ is preserved by the operation $a(x, y)=x \oplus y \oplus 1$. Let us say that a formula $\phi$ is min-affine if there exists a near-affine relation $T \subseteq\{0,1\}^{n}$ such that $\phi$ is of the form

$$
\bigvee_{t \in T}\left(\left(\bigwedge_{i, j: t_{i}=t_{j}=0} x_{i}=x_{j}\right) \wedge\left(\bigwedge_{i, j: t_{i}=0, t_{j}=1} x_{i}<x_{j}\right)\right)
$$

where $x_{1}, \ldots, x_{n}$ are variables; here, the formula $\phi$ is intended to be interpreted over the ordered rationals.

Theorem 3.4. A temporal relation $R \subseteq \mathbb{Q}^{k}$ is preserved by $\mathrm{mx}$ if and only if it is definable by a formula $\phi\left(v_{1}, \ldots, v_{k}\right)$ that is the conjunction of min-affine formulas, each of which is over a subset of $\left\{v_{1}, \ldots, v_{k}\right\}$.

For a tuple $b=\left(b_{1}, \ldots, b_{n}\right) \in \mathbb{Q}^{n}$, we define the min-tuple of $b$ to be the tuple $t=\left(t_{1}, \ldots, t_{n}\right) \in\{0,1\}^{n}$ such that $t_{i}=0$ if and only if $b_{i}$ is the minimum value in $\left\{b_{1}, \ldots, b_{n}\right\}$; note that the min-tuple of a $\mathbb{Q}$-tuple of nontrivial arity always contains at least one entry equal to 0 . Observe also that a tuple $b \in \mathbb{Q}^{n}$ is in the relation defined by a min-affine formula if and only if the min-tuple of $b$ is in $T$.

We remark that we permit the empty conjunction of min-affine formulas, which we consider to be true, and we also permit min-affine formulas where the near-affine relation $T$ is empty, which formulas are considered to be false.

Proof. For the right-to-left direction, it suffices to show that the claim holds in the case that $\phi$ is defined by a single min-affine formula. So suppose that $\phi$ is of the form described above, with respect to $T \subseteq\{0,1\}^{n}$. Let $b=\left(b_{1}, \ldots, b_{n}\right)$, $b^{\prime}=\left(b_{1}^{\prime}, \ldots, b_{n}^{\prime}\right)$ be two tuples in $\mathbb{Q}^{n}$ that satisfy $\phi$. Let $t, t^{\prime} \in T$ be the mintuples of $b, b^{\prime}$, respectively; so, $t, t^{\prime}$ witness that $b, b^{\prime}$ satisfy $\phi$. Let $m, m^{\prime}$ denote the minimum values in the tuples $b, b^{\prime}$, respectively.

We consider two cases.

Case $m=m^{\prime}$. If $t=t^{\prime}$, then the tuple $\operatorname{mx}\left(b, b^{\prime}\right)$ is witnessed to satisfy $\phi$ via the tuple $t=t^{\prime}$; this can be verified by the definition of mx. If $t \neq t^{\prime}$, then we claim that the tuple $\operatorname{mx}\left(b, b^{\prime}\right)$ is witnessed to satisfy $\phi$ via the tuple $t \oplus t^{\prime} \oplus 1$. First, observe that $t \oplus t^{\prime} \oplus 1$ is contained in $T \cup\{(1, \ldots, 1)\}$ by the assumption that this relation is preserved by the operation $a$; since $t \neq t^{\prime}$, it holds that $t \oplus t^{\prime} \oplus 1$ is not equal to $(1, \ldots, 1)$ and is thus contained in $T$. Next, it is straightforwardly verified from the definition of $\mathrm{mx}$ that $\mathrm{mx}\left(b, b^{\prime}\right)$ takes on its minimum value at exactly the 
coordinates where one of $b, b^{\prime}$ takes on its minimum value and the other does not, which are precisely the coordinates where $t \oplus t^{\prime} \oplus 1$ is equal to 0 .

Case $m \neq m^{\prime}$. We assume for the sake of notation that $m<m^{\prime}$. In this case, the minimum entries among all entries in $b$ and $b^{\prime}$ are the entries in $b$ that have value $m$. It follows from the definition of $\operatorname{mx}$ that $\operatorname{mx}\left(b, b^{\prime}\right)$ takes on its minimum value at exactly the coordinates where $b$ does so, and hence the tuple $\operatorname{mx}\left(b, b^{\prime}\right)$ satisfies $\phi$ via the tuple $t \in T$.

For the left-to-right direction, we proceed by induction on the arity $k$ of the relation $R \subseteq \mathbb{Q}^{k}$. The case $k=0$ is verified as follows: if $R$ is empty, then take the empty conjunction; if $R$ is non-empty, then take the conjunction consisting of the single min-affine formula on no variables with $T=\emptyset$. In what follows, we assume that $k>0$. Let $\phi$ be the conjunction of all min-affine formulas that are entailed by $R\left(v_{1}, \ldots, v_{k}\right)$. Suppose that $b=\left(b_{1}, \ldots b_{k}\right) \in \mathbb{Q}^{k}$ satisfies $\phi$. Our goal is to show $b \in R$. Let $t \in\{0,1\}^{k}$ be the min-tuple of $b$.

We claim that there is a tuple $c \in R$ with min-tuple $t$. Let $T \in\{0,1\}^{k}$ be the set of min-tuples of tuples in $R$. It follows directly from (Lemma 28 of [7]) that if $s, s^{\prime} \in T$, then $s \oplus s^{\prime} \oplus 1 \in T \cup\{(1, \ldots, 1)\}$. From this implication, it is straightforward to verify that $T$ is near-affine. Hence, the conjunction $\phi$ contains as a conjunct a min-affine formula $\alpha$ with relation $T$. Since $b$ satisfies $\alpha$, it follows that $t \in T$, from which the claim follows.

If $t=(0, \ldots, 0)$, then $c \in R$ and $b$ are both constant tuples. Since $b$ is equal to $c$ under an automorphism of $(\mathbb{Q} ;<)$, we have $b \in R$. So, we suppose that $t$ contains 1 as an entry; for the sake of notation, we assume that $t$ has the form $(1, \ldots, 1,0, \ldots, 0)$, where the first $m$ entries are 1 ; here, $0<m<k$. We have that $\left(b_{1}, \ldots, b_{m}\right)$ satisfies all min-affine formulas on variables from $v_{1}, \ldots, v_{m}$ which are entailed by $R\left(v_{1}, \ldots, v_{k}\right)$. Hence, by induction, it holds that $\left(b_{1}, \ldots, b_{m}\right) \in$ $\pi_{1, \ldots, m} R$, and that there exists a tuple of the form $\left(b_{1}, \ldots, b_{m}, d\right)$ in $R$, where $d$ is a tuple of length $(k-m)$. We can apply an automorphism to the tuple $\left(b_{1}, \ldots, b_{m}, d\right)$ to obtain a tuple $\left(b_{1}^{\prime}, \ldots, b_{m}^{\prime}, d^{\prime}\right) \in R$ where all entries are positive. Also, by applying an automorphism to $c \in R$, we can obtain a tuple of the form $\left(c_{1}^{\prime}, \ldots, c_{m}^{\prime}, 0, \ldots, 0\right)$ where, for all coordinates $i$ from 1 to $m$, it holds that $c_{i}^{\prime}>b_{i}^{\prime}$. Applying $m x$ to the tuples $\left(b_{1}^{\prime}, \ldots, b_{m}^{\prime}, d^{\prime}\right)$ and $\left(c_{1}^{\prime}, \ldots, c_{m}^{\prime}, 0, \ldots, 0\right)$, one obtains the tuple $\left(\operatorname{mx}\left(b_{1}^{\prime}, c_{1}^{\prime}\right), \ldots, \operatorname{mx}\left(b_{m}^{\prime}, c_{m}^{\prime}\right), \operatorname{mx}\left(d^{\prime}, \overline{0}\right)\right)$, where $\overline{0}$ is a tuple of length $(k-m)$ with all entries equal to 0 , which is equivalent to $b$ under an automorphism.

Example. Observe that the relation $X$ closed under mx presented in Section 2 is defined there as a conjunction of min-affine formulas.

\section{Tractability Results}

In this section, we prove the following theorem.

Theorem 4.1. Let $\Gamma$ be a temporal constraint language preserved by an operation $h \in\{\min , \mathrm{mx}\}$. The problem $\mathrm{QCSP}(\Gamma)$ is polynomial-time decidable.

Let $\Gamma$ be a relational $\tau$-structure. Formulas of the form

$$
Q_{1} v_{1} \ldots Q_{n} v_{n}\left(\psi_{1} \wedge \cdots \wedge \psi_{m}\right)
$$


where each $\psi_{i}$ is of the form $R\left(y_{1}, \ldots, y_{k}\right)$ for $R \in \tau$, and where $Q_{1}, \ldots, Q_{n} \in\{\exists, \forall\}$, will be called quantified constraint formulas (over $\Gamma$ ). Existentially quantified variables are typically (but not exclusively) denoted by $x, x_{1}, x_{2}, \ldots$, and universally quantified variables by $y, y_{1}, y_{2}, \ldots$.

Lemma 4.2. Let $\Gamma$ be a temporal constraint language preserved by an operation $h \in\{\mathrm{min}, \mathrm{mx}\}$. Let $\Phi\left(z_{1}, \ldots, z_{m}\right)=\forall y \exists x_{1} \ldots \exists x_{n} \phi$ be a quantified constraint formula over $\Gamma$ having free variables $\left\{z_{1}, \ldots, z_{m}\right\}$. Let $\Phi^{\prime}\left(z_{1}, \ldots, z_{m}\right)$ be the formula $\exists y \exists x_{1} \ldots \exists x_{n}\left(\phi \wedge \bigwedge_{i \in[m]}\left(z_{i}<y\right)\right)$. If the formula $\Phi$ is satisfiable, then the formulas $\Phi, \Phi^{\prime}$ have the same satisfying assignments over $\Gamma$.

Proof. Observe that for every $q \in \mathbb{Q}$ there exists a value $\alpha(q)$ such that $h(q, p)=$ $h(p, q)=\alpha(q)$ for all $p>q$. Notice that $\alpha(q)$ is injective and preserves $<$.

Let $\Psi$ be the formula $\exists x_{1} \ldots \exists x_{n} \phi$ with free variables $\left\{z_{1}, \ldots, z_{m}, y\right\}$. Assume that $\Phi$ is satisfiable; then, there exists a tuple $\left(g_{1}, \ldots, g_{m}\right) \in \mathbb{Q}^{m}$ such that, for all $d \in \mathbb{Q}$, the tuple $\left(g_{1}, \ldots, g_{m}, d\right)$ satisfies $\Psi$. Clearly, all satisfying assignments of $\Phi$ are satisfying assignments of $\Phi^{\prime}$. We thus need to show that each satisfying assignment of $\Phi^{\prime}$ is a satisfying assignment of $\Phi$.

Let $\left(f_{1}, \ldots, f_{m}\right) \in \mathbb{Q}^{m}$ be a satisfying assignment of $\Phi^{\prime}$. By the definitions of the formulas, there exists a value $b \in \mathbb{Q}$ with $b>f_{i}$ for all $i \in[m]$ such that $\left(f_{1}, \ldots, f_{m}, b\right)$ satisfies $\Psi$. By applying a suitable automorphism to $\left(g_{1}, \ldots, g_{m}\right)$, we may assume that $f_{i}<g_{i}$ for all $i \in[m]$. Now consider $h\left(\left(f_{1}, \ldots, f_{m}, b\right),\left(g_{1}, \ldots, g_{m}, d\right)\right)$ where $d \in \mathbb{Q}$. In the first $m$ coordinates, this tuple is equal to $\left(\alpha\left(f_{1}\right), \ldots, \alpha\left(f_{m}\right)\right)$. In the last coordinate, this tuple is equal to $h(b, d)$; by varying $d$, the value $h(b, d)$ can take on values from every orbit, where here we understand orbit to be with respect to the group consisting of the automorphisms of $(\mathbb{Q},<)$ that fix the values $\alpha\left(f_{i}\right)$. It follows that $\left(\alpha\left(f_{1}\right), \ldots, \alpha\left(f_{m}\right)\right)$ satisfies $\Phi$; since $\alpha^{-1}$ preserves all first-order definable relations, we conclude that $\left(f_{1}, \ldots, f_{m}\right)$ satisfies $\Phi$.

With this lemma in hand, we now prove the theorem. Let $\Gamma$ be a structure that is preserved by min or by $\mathrm{mx}$. We now present an algorithm for $\mathrm{QCSP}(\Gamma)$. For the sake of notation, we assume that the input instance is of the form $\forall y_{1} \exists x_{1} \ldots \forall y_{n} \exists x_{n} \phi$, that is, it exhibits a strict alternation between the two quantifier types. The result for the general case can be obtained, for example, by considering an algorithm that inserts dummy variables/quantifiers into an arbitrary instance of QCSP $(\Gamma)$ to massage it into the described form, and then passes to the algorithm that we describe. We make use of the fact that $\operatorname{CSP}(\Gamma)$ can be decided by the algorithms given in 7 ; indeed, those algorithms describe how to compute satisfying assignments in the event that they exist.

We now discuss the correctness of this algorithm. The algorithm maintains the invariant that, at the beginning of each loop, the formula $\Psi_{i}\left(y_{1}, x_{1}, \ldots, y_{i}, x_{i}\right)$ is equivalent to the formula $\forall y_{i+1} \exists x_{i+1} \ldots \forall y_{n} \exists x_{n} \phi$. (By equivalent, we mean that the formulas have the same satisfying assignments over $\Gamma$.) This is certainly true for $i=n$ by the initialization of $\Psi_{n}$.

We now consider the behavior of the algorithm for an execution of the loop. If the formula $\Phi_{i}^{\prime}$ is not satisfiable, it follows that the formula $\Phi_{i}$ is not satisfiable (as any satisfying assignment for $\Phi_{i}$ is clearly one for $\Phi_{i}^{\prime}$ ), and hence that the original sentence is false; the algorithm hence reports "False" correctly.

In the case that $\Phi_{i}^{\prime}$ is found to be satisfiable, consider first the case that the satisfying assignment $w$ for $\Phi_{i}^{\prime}$ does not satisfy $\Phi_{i}$. It follows by Lemma 4.2 that $\Phi_{i}$ 


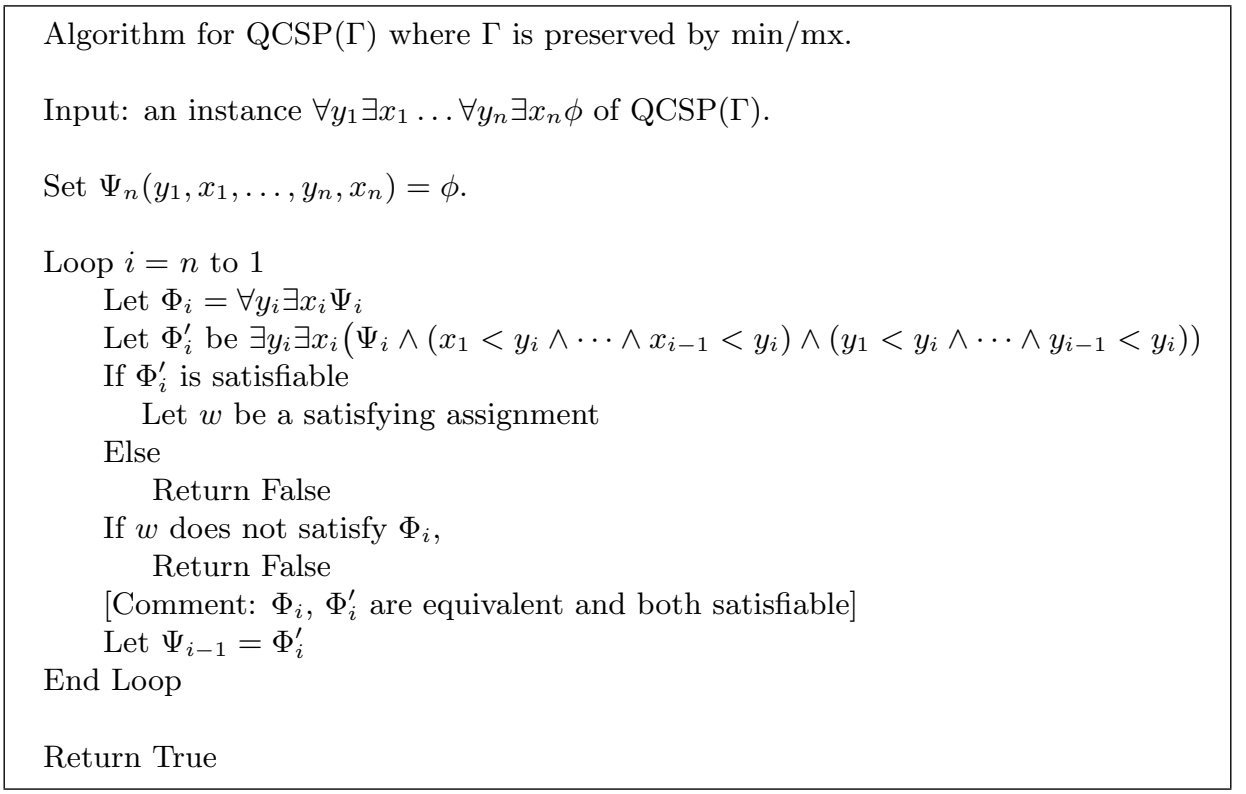

is not satisfiable, and hence that the original sentence is false; the algorithm hence reports "False" correctly.

In the case that the assignment $w$ does satisfy $\Phi_{i}$, by Lemma 4.2 , the formulas $\Phi_{i}, \Phi_{i}^{\prime}$ are equivalent. Consequently, when $\Psi_{i-1}$ is set equal to $\Phi_{i}^{\prime}$, by the definition of $\Phi_{i}$ and the fact that the invariant held at the beginning of the loop, the invariant will hold for the next execution of the loop. In the case that $i=1$, if the loop does not return false, by the given argumentation, we have that both $\Phi_{1}$ and $\Phi_{1}^{\prime}$ are true, and hence that the original sentence is true.

\section{Discussion}

The polynomial-time algorithms for the QCSP of temporal constraint languages that are preserved by min or preserved by $\mathrm{mx}$ that we have seen in this paper properly strengthen previously known algorithmic results for the QCSP:

- In 12, it has been shown that instances of the QCSP where all constraints are of the form $x \geq y_{1} \vee \cdots \vee x \geq y_{k}$ can be solved in polynomial time. All such constraints are preserved by min (this follows immediately from the syntactic characterization that we gave for temporal relations that are preserved by min).

- In 4, it has been shown that the QCSP for all temporal constraint languages preserved by min is in NP.

There are (up to duality) two more temporal constraint languages whose CSP is maximally tractable [7. One of them is characterized by a certain binary polymorphism called ll. The tractability for the CSP for ll-closed languages has been shown in [8]. The QCSP for ll-closed languages is coNP-hard [6], so this is an instance where maximal tractability doesn't transfer to the QCSP. There is the temporal constraint language that contains all relations preserved by the binary operation 
mi (for a definition, see [7]). In this case, the tractability of the QCSP is open. In fact, already the following is open.

Question 5.1. Is $\mathrm{QCSP}((\mathbb{Q} ;\{(x, y, z) \mid x=y \Rightarrow y \leq z\}))$ in $\mathrm{P}$ ?

The relation defined by $x=y \Rightarrow y \leq z$ is indeed preserved by mi (see [7]). Finally, the temporal languages preserved by a constant operation have a tractable CSP [7. As shown in previous work [12, 11, the QCSP for such languages may be contained in LOGSPACE, be NLOGSPACE-complete, be P-complete, be NPcomplete, or be PSPACE-complete.

Another field of open problems is related to the question how bad the complexity of the QCSP becomes when we are outside the maximal tractable classes. From maximal tractability of the CSP we only obtain NP-hardness, but very often the problem might become even PSPACE-hard. Very often, questions in this context lead to the following notorious open problem.

Question 5.2. Is $\operatorname{QCSP}((\mathbb{Q} ;\{(x, y, z) \mid x=y \Rightarrow y=z\}))$ PSPACE-complete?

We only know that this QCSP is coNP-hard [6].

Postprint note: D. Zhuk and B. Martin solved Question 5.2 [22].

\section{REFERENCES}

[1] B. Aspvall, M. F. Plass, and R. E. Tarjan. A linear-time algorithm for testing the truth of certain quantified boolean formulas. Information Processing Letters, 8(3):121-123, 1979.

[2] L. Barto and M. Kozik. Constraint satisfaction problems of bounded width. In Proceedings of the Annual Symposium on Foundations of Computer Science (FOCS), pages 595-603, 2009.

[3] M. Bodirsky. Complexity classification in infinite-domain constraint satisfaction. Memoire d'habilitation à diriger des recherches, Université Diderot - Paris 7. Available at arXiv:1201.0856, 2012.

[4] M. Bodirsky and H. Chen. Collapsibility in infinite-domain quantified constraint satisfaction. In Proceedings of Computer Science Logic (CSL), Szeged, Hungary, 2006.

[5] M. Bodirsky and H. Chen. Relatively quantified constraint satisfaction. Constraints, 14(1):315, 2009.

[6] M. Bodirsky and H. Chen. Quantified equality constraints. SIAM Journal on Computing, 39(8):3682-3699, 2010. A preliminary version of the paper appeared in the proceedings of LICS'07.

[7] M. Bodirsky and J. Kára. The complexity of temporal constraint satisfaction problems. Journal of the ACM, 57(2):1-41, 2009. An extended abstract appeared in the Proceedings of the Symposium on Theory of Computing (STOC'08).

[8] M. Bodirsky and J. Kára. A fast algorithm and Datalog inexpressibility for temporal reasoning. ACM Transactions on Computational Logic, 11(3), 2010.

[9] F. Börner, A. A. Bulatov, H. Chen, P. Jeavons, and A. A. Krokhin. The complexity of constraint satisfaction games and QCSP. Information and Computation, 207(9):923-944, 2009.

[10] P. J. Cameron. The random graph. R. L. Graham and J. Nešetřil, Editors, The Mathematics of Paul Erdös, 1996.

[11] W. Charatonik and M. Wrona. Quantified positive temporal constraints. In Proceedings of CSL, pages $94-108,2008$.

[12] W. Charatonik and M. Wrona. Tractable quantified constraint satisfaction problems over positive temporal templates. In LPAR, pages 543-557, 2008.

[13] H. Chen. The computational complexity of quantified constraint satisfaction. Ph.D. thesis, Cornell University, August 2004

[14] H. Chen. Meditations on quantified constraint satisfaction. In Logic and Program Semantics - Essays Dedicated to Dexter Kozen on the Occasion of His 60th Birthday, Lecture Notes in Computer Science 7230. Springer, 2012. 
[15] N. Creignou, S. Khanna, and M. Sudan. Complexity Classifications of Boolean Constraint Satisfaction Problems. SIAM Monographs on Discrete Mathematics and Applications 7, 2001.

[16] N. Creignou, P. G. Kolaitis, and H. Vollmer, editors. Complexity of Constraints - An Overview of Current Research Themes [Result of a Dagstuhl Seminar], volume 5250 of Lecture Notes in Computer Science. Springer, 2008.

[17] T. Feder and M. Y. Vardi. The computational structure of monotone monadic SNP and constraint satisfaction: a study through Datalog and group theory. SIAM Journal on Computing, 28:57-104, 1999.

[18] W. Hodges. A shorter model theory. Cambridge University Press, Cambridge, 1997.

[19] P. G. Jeavons and M. C. Cooper. Tractable constraints on ordered domains. Artificial Intelligence, 79(2):327-339, 1995.

[20] M. Karpinski, H. K. Büning, and P. H. Schmitt. On the computational complexity of quantified Horn clauses. In Proceedings of CSL, pages 129-137, 1987.

[21] D. Macpherson. A survey of homogeneous structures. Discrete Mathematics, 311(15):15991634, 2011.

[22] D. Zhuk and B. Martin. The complete classification for quantified equality constraints. CoRR, abs/2104.00406, 2021.

Institut für Algebra, TU Dresden, Germany

Email address: manuel.bodirsky@tu-dresden.de

Department of Computer Science, Birkbeck, University of London, Malet Street, London WC1E 7HX, United Kingdom

Email address: hubie@dcs.bbk.ac.uk

Theoretical Computer Science Department, Jagiellonian University, Poland

Email address: michal.wrona@uj.edu.pl 